\title{
Bangladesh: Using strong evidence and strategic collaboration to increase access to menstrual regulation with medication
}

Nancy Termini LaChance

Population Council

Sharif M.I. Hossain

Population Council

Follow this and additional works at: https://knowledgecommons.popcouncil.org/departments_sbsr-rh

Part of the Demography, Population, and Ecology Commons, Family, Life Course, and Society Commons, International Public Health Commons, Maternal and Child Health Commons, Obstetrics and Gynecology Commons, and the Women's Health Commons How does access to this work benefit you? Let us know!

\section{Recommended Citation}

Termini LaChance, Nancy and Sharif M.I. Hossain. 2017. "Bangladesh: Using strong evidence and strategic collaboration to increase access to menstrual regulation with medication," STEP UP Case Study. Dhaka: Population Council. 


\section{CASE STUDY}

NOVEMBER 2017

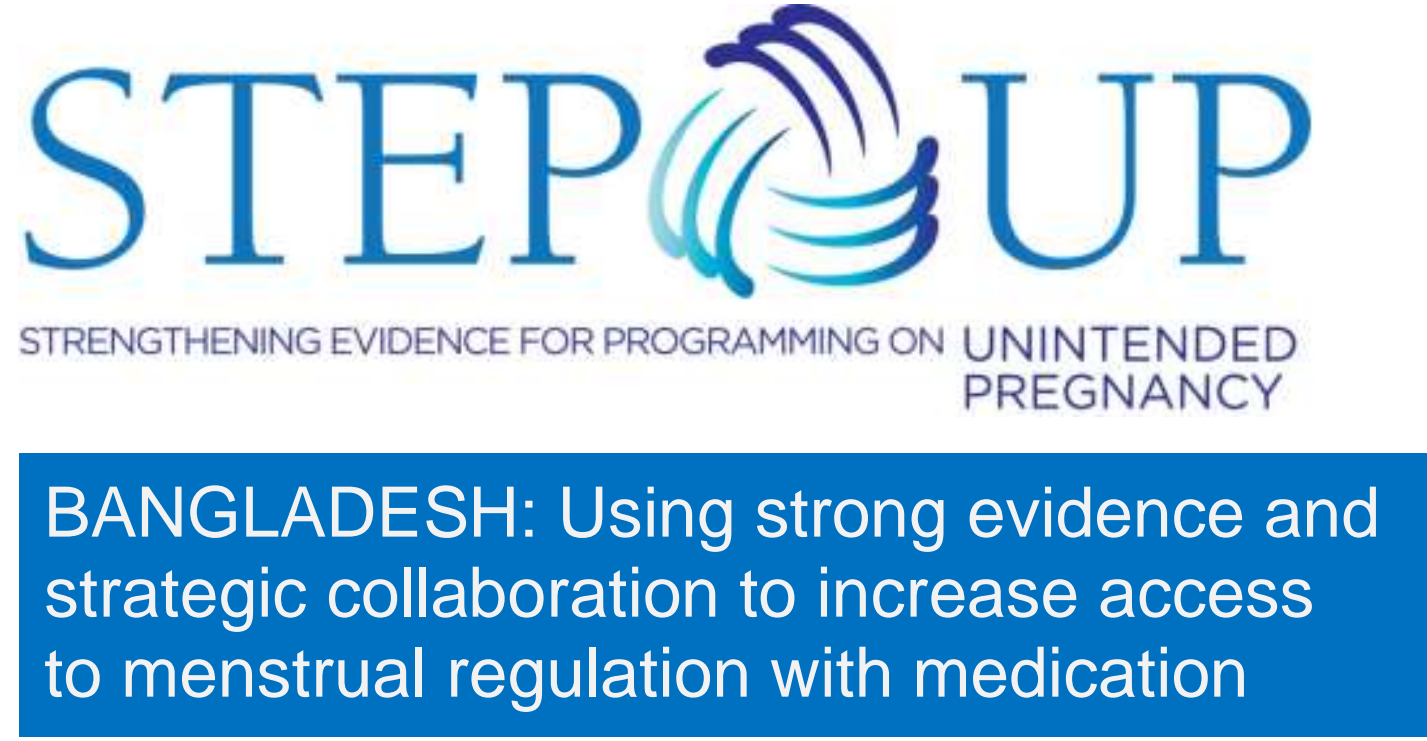

FROM EVIDENCE TO IMPACT: A look at how STEP UP research was used to impact policies and programmes

The goal of family planning and reproductive health operations research is not merely to generate evidence but also to inform policies and programmes to solve the problems of women's access to and quality of services. Yet the crucial step of ensuring the utilization of that evidence often receives inconsistent or inadequate attention. There is relatively little monitoring and reporting on whether and to what effect project results are utilized, or on the nature, process, and efficacy of the strategies employed to achieve this.

The aim of this case study is to present an activity of the STEP UP research programme consortium that resulted in successful evidence utilization. This is to both demonstrate the positive impact STEP UP is having on family planning and reproductive health policies and programmes, as well as to document the process by which this was achieved so as to inform future research of successful strategies and lessons learned.

\section{SUMMARY}

Through close cooperation with the Ministry of Health and Family Welfare (MoH\&FW) of the People's Republic of Bangladesh, and other partners, STEP UP generated strong evidence and cultivated ongoing collaboration that contributed to policy changes and programme expansions. These changes are increasing women's access to menstrual regulation with medication (MRM) in Bangladesh, and include:

- Approval of MRM services introduction in the national family planning program

- Approval of MRM scale up nationwide in public facilities, and creation of strategic plan for scale up;

- Development of national MRM service delivery guidelines and training curriculum;

- MRM combination drugs locally manufactured for first time;

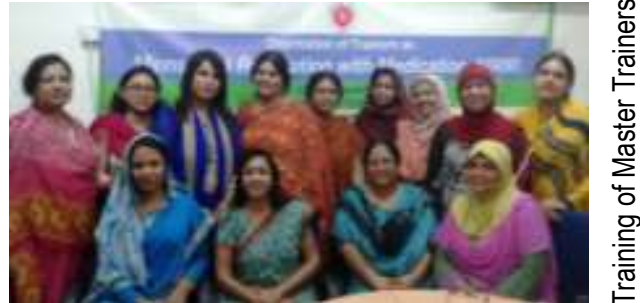

- Memorandum of Understanding signed with the Director General of Family Planning to assist in scale up MRM services to nearly 4,000 health facilities;

- Development of a core group of 13 master trainers and trained service providers in MRM service provision (108 physicians and 126 family welfare visitors from Maternal and Child Welfare Centres (MCWCs); and

- Successful service expansion to 70 urban MCWCs and in all urban health and family welfare centres of two districts 


\section{INTRODUCTION}

Despite the availability of effective modern contraceptive methods in Bangladesh, the number of unsafe abortions rises each year, increasing risk of maternal morbidity and mortality. ${ }^{1,2}$

Menstrual regulation (MR) is an interim method of safely establishing non-pregnancy after a missed period, regardless of whether conception has occurred or not, and is usually done without a pregnancy test. Its availability helps to decrease the number of women who have unsafe abortions - and consequently, decreases maternal mortality and morbidity. ${ }^{3}$ Indeed, effective implementation of the MR policy in Bangladesh has contributed to a reduction in maternal mortality from $24 \%$ to $11 \%$ between 1976 and $2005 .{ }^{4}$ In Bangladesh, MR is typically conducted using manual vacuum aspiration (MVA), a surgical method.

However, MR using MVA carries risks of social stigma and significant post-MVA complications if service quality is poor or unhygienic. Despite the fact that MR using MVA is a safe and legal service that is widely available in most public health facilities, approximately 650,000 women each year are unable to access this service - prompting them to resort to more discreet but illegal induced abortions, most of which are unsafe. ${ }^{2}$ Addressing the barriers that prevent women from using MR services would reduce illegal and unsafe abortion and the associated mortality and morbidity.

Menstrual regulation with medication (MRM) is an important tool for achieving non-pregnancy after a missed period. MRM is a safe, WHO-approved, non-invasive alternative to MVA. The most effective drug formulation for MRM is a Misoprostol-Mifepristone combination.

However, MRM is not yet widely available in Bangladesh. By increasing access to this simple and safe MR option, women's morbidity due to MR and unsafe abortion in Bangladesh could be greatly decreased.

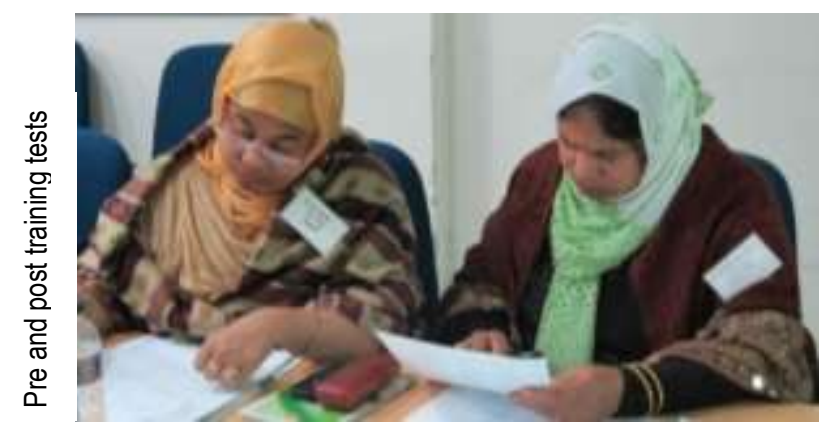

Menstrual regulation with medication (MRM) is a safe, WHO-approved, non-invasive

alternative to MVA that is approved in

Bangladesh but not yet widely available. By increasing access to this simple and safe $M R$ option, women's morbidity due to MR and unsafe abortion could be greatly decreased.

\section{THE PROJECTS}

STEP UP partners in Bangladesh have worked to understand the barriers to MRM access and identify possible solutions. Our projects have explored (1) why women undergo induced and/or unsafe abortions and what barriers they face to accessing legal MR, and (2) the feasibility of introducing MRM in Bangladesh as a solution, and the acceptability of providing MRM using the Mifepristone-Misoprostol drug combination in urban and rural public health facilities.

Understanding unintended pregnancy in Bangladesh: Country Profile: STEP UP conducted an extensive review and critical analysis of literature on existing MR and abortion policies and programmes in Bangladesh, seeking to understand why and how women undergo unsafe abortions and why they do not obtain MR. Relevant local research-based organisations were consulted, pertinent documents were collected, and PubMed/MEDLINE, HINARI, JSTOR and Google Scholar were searched. ${ }^{5}$

icddr,b has also developed a stakeholder analysis which provides information about factors that contribute to pregnancy termination in Bangladesh and about a range factors that contributed to the approval process of MRM in Bangladesh. ${ }^{6}$
Assessing the feasibility and acceptability of introducing MRM in Bangladesh: In collaboration with the Directorate General of Family Planning (DGFP) and the World Health Organisation, STEP UP partners tested the feasibility and acceptability of introducing MRM using the Mifepristone-Misoprostol drug combination in urban and rural public health facilities. An 18-month operations research study was conducted from January 2012 to June 2013. Health facilities were selected with high numbers of MR clients and available trained service providers. ${ }^{1}$ All women who visited the 13 health facilities for MR services 
from October 2012 -- May 2013 were invited to participate in the study. All women were counseled by trained service providers about the advantages, disadvantages, and side effects of MR and given a choice between MVA and MRM to regulate menstruation.

\section{KEY STUDY FINDINGS}

Understanding barriers to women's access to $M R$ and recourse to unsafe abortion

- MR services (MVA) are available at the majority of public facilities at district, sub-district, and union levels, as well as at some private and NGO facilities.

- Women are deterred from seeking MR services due to distance to the facility, unofficial fees for the service, lack of privacy and risk of stigma, and negative provider attitudes. ${ }^{5}$

- As of 2011, 10,600 doctors and 7,200 paramedics (mainly family welfare visitors) had been trained to provide MR services through MVA, yet a minority of the providers trained to provide MR actually do so,, 7

- $26 \%$ of women seeking MR are rejected by providers. Most providers indicated that the women had exceeded the then-legal time limit of 10 weeks since their last menstrual period, but many rejected women for discriminatory reasons.

- Many of these providers refuse to provide MR services because of personal feelings, including: if the woman is childless $(20 \%)$, if they felt she was too young $(12 \%)$ if she is unmarried, widowed, or divorced (8\%) or if she lacks consent from her husband or guardian, although this is only legally required for minors or the mentally handicapped $(7 \%) .5,8$

- Few women (11\%) obtain MR free of charge, despite the fact that public-sector MR is supposed to be available at no cost. ${ }^{5}$

- Adolescent girls - especially those who are unmarried - are at higher risk of unsafe abortion One study indicated that the risk of abortion for unmarried adolescents was 35 times greater than among those who are married. This may be due to high stigma against unmarried pregnancy, or to inadequate adolescent awareness of reproductive health resources including MR. Sex workers are also at higher risk of unsafe abortion.5,9
Testing the feasibility and acceptability of introducing MRM into

rural and urban public health facilities

- Nearly all providers identified the last menstrual period and $70 \%$ took a menstrual history. The overall quality of services provided at these health facilities was high.

- One in five clients experienced a side effect (commonly nausea) after taking Mifepristone (first dose), although less than half of these women required medication or further treatment for the side effects.

- Almost two-thirds of women preferred MRM to MVA.

- Nearly all women who chose MRM (97\%) reported that they would suggest MRM to their friends and relatives: $95 \%$ would refer a friend to the same facility. Virtually all women receiving MRM reported being satisfied/very satisfied with the quality of care.

- The vast majority $(92 \%)$ of women returned to the facility after 14 days for the examination to confirm procedure completion. ${ }^{1}$

The study suggested that it is safe and feasible to introduce MRM in Bangladesh in rural and urban public health facilities.

\section{MAKING AN IMPACT}

\section{Influencing policy and programme improvements}

The legalization, introduction, and expansion of MRM in Bangladesh has been made possible by the collaborative efforts of many local, national, and international stakeholders, of which STEP UP is one. Specific to our efforts, focused stakeholder engagement and utilization of study findings contributes to several impacts:

\section{MRM approved for introduction and scale-up in national family planning programme}

Findings of the study were presented at the 61st National Technical Committee (NTC) meeting and it was subsequently approved by the NTC and Drug Administration that the combination MRM drug (Mifepristone-Misoprostol) be introduced into the national family planning programme. It was approved in this meeting that initial introduction be initiated by phases, starting in urban areas. ${ }^{10} \mathrm{lt}$ was also requested by DGFP that STEP UP provide technical assistance during the scale up phases to ensure availability of MRM services through trained health care providers. 
Memorandum of Understanding signed to scale up MRM nationwide after initial introduction

Based on discussions during the NTC meeting, a tripartite Memorandum of Understanding (MOU) was signed between the Directorate General of Family Planning (DGFP) under the MoH\&FW, Bangladesh, and STEP UP partners (Population Council and MSI) to scale up MRM throughout Bangladesh.

In this MOU, STEP UP agreed to provide technical assistance for the scaling up of MRM services to all (nearly 4,000) Maternal and Child Welfare Centres (MCWCs) and Union Health and Family Welfare Centres (UH\&FWCs), to support the implementation process, and to assist the DGFP to ensure the availability of the required commodities. Subsequently, a project advisory committee (PAC) and technical group were formed to support STEP UP in carrying out this agreement.

\section{National service delivery guidelines and training} curriculum developed

As agreed in the MOU, the DGFP formed a technical committee (including the DGFP, DGHS, Obstetrical and Gynecological Society of Bangladesh, RH STEP, Marie Stopes Bangladesh, Ipas, and BAPSA) to develop national service delivery guideline for MRM. STEP UP worked within the technical committee as well as with local experts to develop the service delivery guidelines and a training curriculum for MRM service providers.

These guidelines and curricula were finalized and approved by the PAC, translated into Bengali, and are being used to provide consistent information to different cadres of service providers.

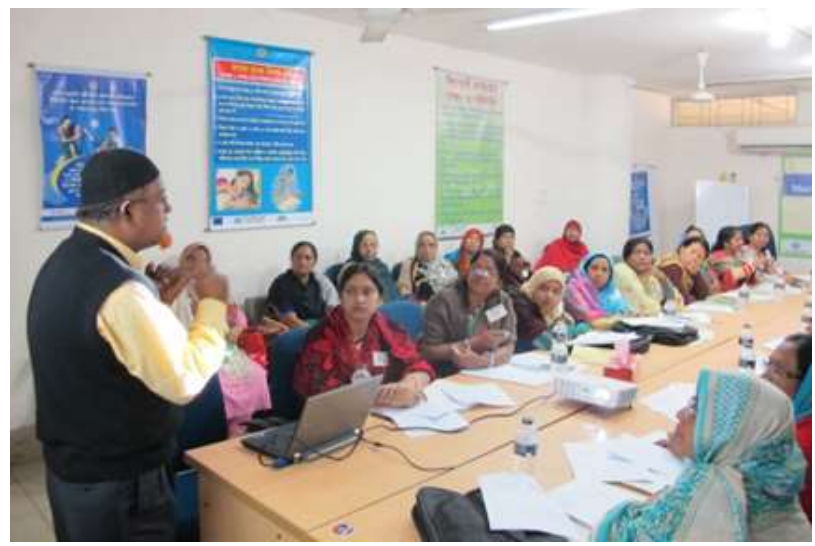

Dr. Farid Uddin Ahmed, Assistant Director, MCH-Service Unit, DGFP in a training session
Strategic plan for MRM service scale-up created STEP UP assisted the DGFP in the development of a strategic plan for the vertical expansion of MRM services in the nearly 4,000 MCWCs and UH\&FWCs. The DGFP will carry out this expansion starting with the incorporation of MRM services into their $5^{\text {th }}$ HNP sector programme. The scale up programme has been completed in all 70 MCWCs and two districts of Khulna Division.

Master Trainers identified and trained

One of the major objectives of MRM scale-up activities was to develop a national pool of Master Trainers (MTs) on MRM who can be utilized during the nationwide services expansion. The MTs were selected by the PAC and were from DGFP, DGHS, OGSB, MSB and Population Council. All MTs were physicians and had previous extensive experience on training on MR service provision. A total of 13 MTs were trained.

Urban service providers trained in MRM provision While testing the feasibility of providing MRM services to women in public health facilities, STEP UP trained all eligible providers in 70 urban Maternal and Child Welfare Centres in safe provision of MRM services. A total of 108 physicians and $126 \mathrm{FWVs}$ were trained.

\section{MRM combination drugs supplied to 70 MCWCs} With support from WHO, Marie Stopes supplied MRM combinations drugs to all 70 MCWCs. A total of 12,000 combinations drugs were supplied, a one-year supply.

\section{MRM training expanded into rural areas}

Further, STEP UP organized an orientation and training programmes in Khulna Division to underpin the DGFP's scale-up of services according to the strategic plan. Sixty (60) programme managers of 10 districts of Khulna Division received training in MRM service provision and 28 medical officers for maternal and child health and family planning $(\mathrm{MOMCH})$ were trained as trainers to enable subsequent provider training (which was supported by two Master Trainers from the Ministry of Health and Family Welfare).

Training on MRM was further expanded to Family Welfare Visitors (FWVs) and female Sub Assistant Community Medical Officers (SACMOs) of Khulna and Jessore District. Training was conducted by a government Master Trainer and two medical officers (MOMCH) who had participated in the Training of Trainers programme. A total of 73 FWVs from UH\&FWCs and 6 female SACMOs in both districts. 


\section{MRM combination drugs locally manufactured for first time}

After the approval of MRM drugs by the Directorate general of Drug Administration (DGDA), several local pharmaceutical companies in Bangladesh started manufacturing the Misoprostol/Mifepristone combination drug. Now, it is manufactured by several companies.

This change stands to promote women's access to MRM commodities by making drugs more readily available to local providers, reducing stock outs, and improving the supply chain.

\section{Who will benefit?}

\section{- Women in need of safe, accessible MRM} services: Having access to quality MRM services may help women in Bangladesh to avoid resorting to invasive procedures like MVA or unsafe methods of pregnancy terminations. This stands to help prevent women's illness, injury, and even death. Further, before the addition of MRM to the method mix, only MVA was available. Adding more options gives women the freedom to choose the method that is right for them.

- Public health facilities: If women increasingly use MRM instead of MVA or unsafe methods of pregnancy termination, fewer women may need to be treated for complications from these procedures, thus reducing the burden placed upon clinics that may be overworked and underfunded. Furthermore, by increasing the number of providers who can provide MRM, improving their training, and improving the availability of supplies, public health facilities may provide better services more reliably.

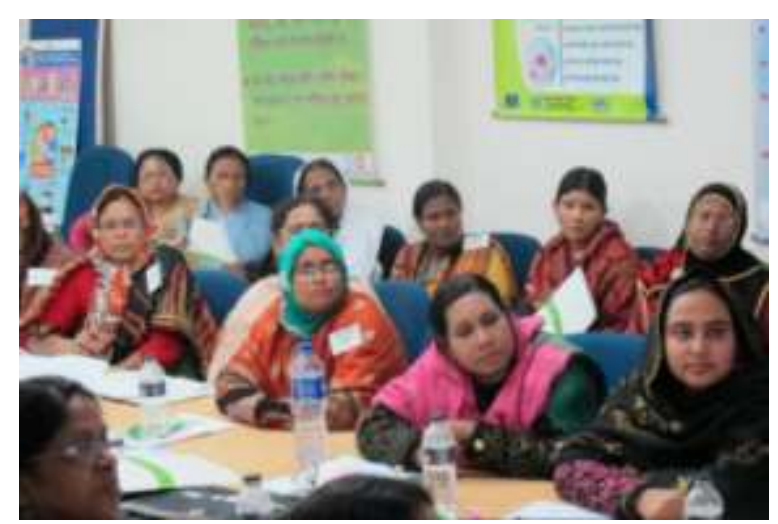

FWVs training session
HOW WAS IMPACT ACHIEVED?

\section{SOME KEY LESSONS LEARNED}

\section{Close collaboration with government and professional societies}

Critical to STEP UP's impact in Bangladesh has been a close and ongoing collaboration with both government ministries as well as with professional organisations. Importantly, the project team sought to ensure these individual relationships remained positive, cooperative, reliable, and were productive rather than obstructive. STEP UP activities also have had the support and participation from not only the broad membership of these groups but from individuals in leadership roles.

The Ministry of Health and Family Welfare, specifically the Dr. Nur Hossain Talukder (Director General, DGFP), and Dr. Mohammed Sharif (Director, MCH Services Unit, DGFP) took an active role in organizing and implementing the MRM training programme. This included the formation of a technical working group, hosting the training programme and providing resource persons, and the DGFP inaugurating and closing each training session.

STEP UP also collaborated closely with the Obstetrical and Gynaecological Society of Bangladesh (OGSB). The OGSB played an important role in developing the service delivery guidelines, reviewing information education and communication (IEC) materials, and providing training to the service providers. Former OGSB President Dr. Rashwan Ara and Secretary General Dr. Farhana Dewan actively participated in developing the service delivery guideline, selection of Master Trainers and providing training to Master Trainers. Furthermore, former president of the OGSB Dr. Latifa Samsuddin attended each training session.

When the STEP UP team proposed policy adoption before the NTC, it was done in collaboration with local policy makers and programme managers. This dynamic of partnership, inclusivity, and responsiveness to identified local needs was key in successful adoption of policies. 
Strong project partnership featuring a range of expertise and resources

Marie Stopes Bangladesh (MSB), the Population Council, and OGSB brought unique strengths to a productive partnership, which was significant in helping to promote MRM policy adoption. That MSB is a service delivery agency was valuable in helping to make the study's recommendations feasible and acceptable to the MOHFW. MSB was able to facilitate clinical training sessions, procure and supply the necessary MRM drugs to the DGFP, and serve as referral centres to provide backup support to referred MRM clients of government facilities for management of side effects or complications.

Population Council research, in addition to data gathered by icddr,b, contributed strong evidence that helped to underpin the merit of the policy and programme changes made to support MRM service provision. The Council also helped to lead in the design, organisation, and implementation of the overall MRM scale up activities, logistical support and knowledge management, including the development of two databases of service providers posted to MCWCs, aiding in effective communication for selection of participants in MRM training.

\section{Sound evidence that reflected a receptive public, engaged service providers, and supportive policy environment}

Studies carried out by STEP UP and others generated strong evidence indicating that women vastly preferred MRM over MVA - especially the ease, safety, and privacy of MRM. ${ }^{1}$ Furthermore, although some service providers decline to provide MRM, the majority indicated that being able to offer MRM would enable them to better serve women for whom MVA was an inappropriate option, or when MVA supplies were limited. ${ }^{11}$ They expressed excitement about MRM as a welcome, safer, in-demand service that they wanted to be able to offer women. ${ }^{12}$ Providers and clients alike perceived MRM as a non-invasive, more suitable, technologically-simple, and easy-to-use alternative method to MVA. ${ }^{6}$

In addition, linking project activities to evidence and recommendations from $\mathrm{WHO}$ - as a respected and globally-acknowledged authority on the issue - helped to affirm STEP UP's findings and recommendations as credible among providers and policymakers.

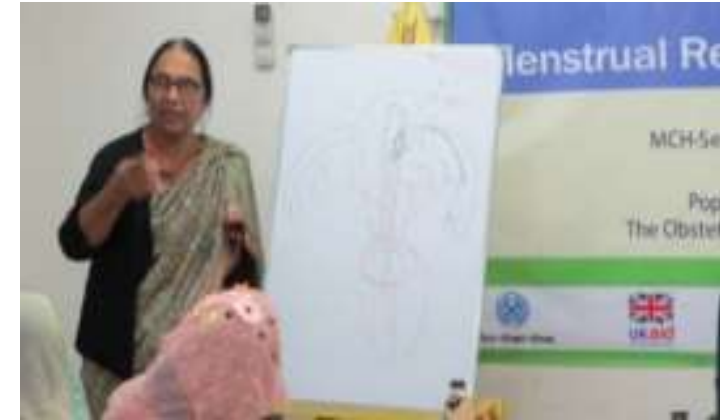

Professor Dr. Latifa Samsuddin, immediate past President, OGSB conducting session

The DGFP was keen to introduce an alternative to MVA, reflected in their commitment to support STEP UP research and formalizing an MOU with Population Council and MSB. Their support was continually borne out throughout STEP UP's research and training processes; the $\mathrm{MCH}-S e r v i c e s$ unit has taken active roles in organizing and implementing the MRM training program, has formed a project advisory committee to guide, monitor and supervise the programme activities, has formed a technical working group to guide development of guidelines and IEC materials, and hosts and staffs the MRM training programmes.

\section{DISCUSSION}

Achieving impact through research uptake hinged on several strategies that enabled the project partners to identify and pursue the relevant local mechanisms for policy adoption. These strategies included:

- Close collaboration with government and professional societies

- Strong project partnership featuring a range of experience and resources

- Strong evidence generated that reflected a receptive public, open-minded service providers, and supportive policy environment

It was important for STEP UP to underpin the above efforts by focusing on the dynamics with which they engaged policymakers. By cultivating sensitive interpersonal dynamics that paid attention to policy maker priorities, concerns, obstacles, and opportunities, STEP UP partners were able to build trust, strategically frame recommendations and their intended outcomes in positive terms that focused on shared goals, prioritize participation of policymakers and managers in evidence gathering, and generally ensure that personal relationships with stakeholders and policymakers remained pleasant, cooperative, and reliable. 


\section{CONCLUSION}

Although nearly all contraceptive methods are approved and available in Bangladesh, each year unintended pregnancies compel women to resort to unsafe methods of pregnancy termination. MR is a procedure that uses MVA to safely establish nonpregnancy after a missed period, but is not suitable for all women. MVA can be unsafe when the quality of service is poor, administered by inadequately trained service providers in unhygienic settings. Menstrual Regulation with Medication (MRM) is a safe, effective, low-cost alternative to MVA that is vastly preferred by both women and providers.

The most effective drug formulation for MRM is a Misoprostol-Mifepristone drug combination. Previous to STEP UP's activities, the drug Misoprostol had been available in Bangladesh for several non-MRM uses. However, Mifepristone was not approved as it was branded as abortifacient. Through collaborative advocacy efforts and strong evidence, the combined drug was approved for use in MRM services, is being locally manufactured, and is being used in nationwide scale up of MRM service delivery.

Now that it is clear that MRM is acceptable and effective in Bangladesh, has been legalized and folded into the national FP program, and is being scaled up nationwide, ongoing observation and studies are still needed. It is important to understand whether the efficacy and acceptability of MRM remains the case when services are delivered at scale on a national level. Follow-up studies should gather data on who is seeking services, whether they are doing it correctly, how frequently they are doing it, what complications they face (if any), are whether they are getting enough attention if there is a complication.

Additionally, although the government is coordinating nationwide scale up in public facilities, we must consider the role that the private sector plays in women's access to MRM, especially considering that the government has little control over the drugs sold in pharmacies. Although the drugs are not approved for sale over-the-counter, pharmaceutical companies are aggressively marketing Misoprostol to pharmacists, despite the fact that they may not be properly trained and the fact that Misoprostol is less effective for MRM than the combination drug.

\section{Suggested citation:}

Nancy Termini LaChance, Sharif Mohammed Ismail Hossain. 2017.

"Using strong evidence and strategic collaboration to increase access

to menstrual regulation with medication," STEP UP Case Study,

November 2017. Dhaka: Population Council.

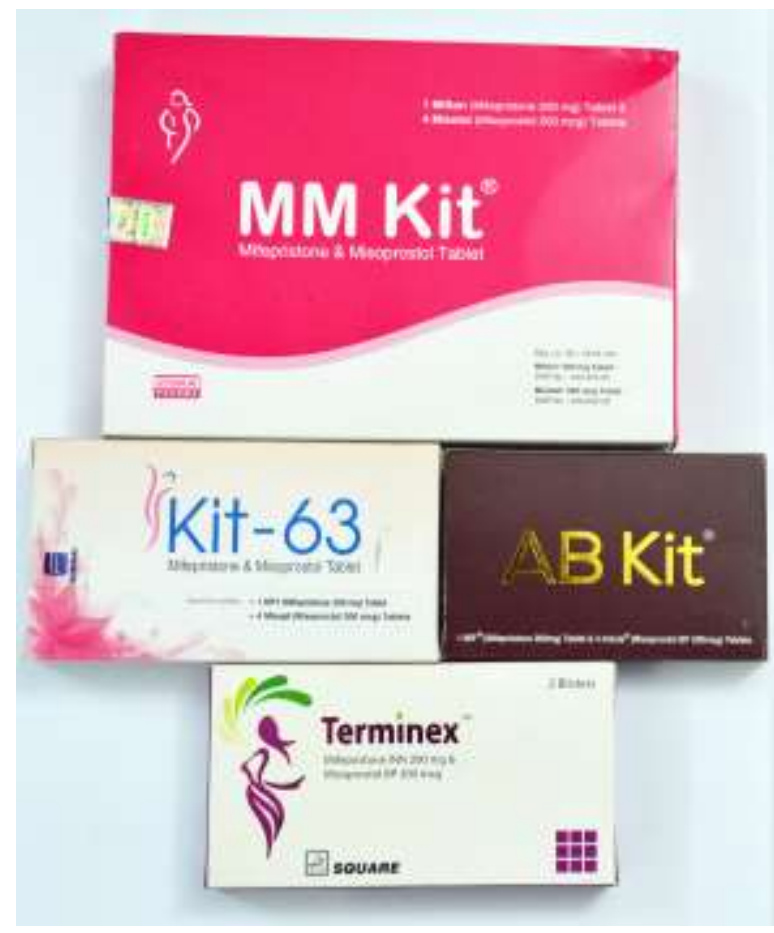

MRM kit available in Bangladesh

\section{REFERENCES}

1. Hena IA et al. 2013. Introducing Medical Menstrual Regulation in Bangladesh: MRM Final Report. Dhaka, Bangladesh: Population Council.

2. Singh, $S$ et al 2012. The Incidence of Menstrual Regulation Procedures and Abortion in Bangladesh. International Perspectives on Sexual and Reproductive Health, 38(3): 122-132, doi: 10.1363/3812212.

3. Reproductive Health Matters, February 2015, Volume 22, Issue 44 Supplement 1, p1-143.

4. Chowdhury ME et al. 2009. Causes of maternal mortality decline in Matlab Bangladesh, Journal of Health, Population and Nutrition, 27(2):108-123.

5. Huda, FA et al. 2013. Understanding Unintended Pregnancy in Bangladesh: Country Profile Report. STEP UP Research Report. Dhaka: icddr,b.

6. Huda FS, Afrin S, Sarker BK, Mahmood HR, Alam A. 2017. Introduction and approval of menstrual regulation with medication in Bangladesh: A stakeholder analysis. STEP UP Research Report. Dhaka: icddr,b.

7. Sexual and Reproductive Health and Rights Consortium. MR services: performance statistics, in: Health and Rights, Dhaka: Bangladesh, Sexual and Reproductive Health and Rights Consortium, No. 4, Issue 4, 2011, p. 4

8. Vlassoff $\mathrm{M}$ et al., Menstrual Regulation and Postabortion Care in Bangladesh: Factors Associated with Access to and Quality of Services, New York: Guttmacher Institute, 2012.

9. Ahmed MK et al. Factors associated with adolescent abortion in a rural area of Bangladesh. Tropical Medicine and International Health 2005 February; 10 (2):198-205.

10. Bangladesh DGFP. 2013. Proceedings of the $61^{\text {st }}$ meeting of the National Technical Committee (NTC) held on 26 November, 2013. Dhaka: DGFP.

11. Yasmin $R$ et al. 2015. Increasing access to safe menstrual regulation services in Bangladesh by offering medical menstrual regulation. Reproductive Health Matters 2015; Supplement (44):67-74

12. Alam A et al. 2013. Acceptability and feasibility of mifepristone-misoprosto for menstrual regulation in Bangladesh. International Perspectives on Reproductive Health, 2013, 39(2):79-87.

13. DGFP, Population Council and Marie Stopes. 2015. Memorandum of Understanding. Dhaka: DGFP. 